Article

\title{
First Insights on Organic Cosolvent Effects on FhuA Wildtype and FhuA $\Delta 1-159$
}

\section{Stefanie-Joana Tenne and Ulrich Schwaneberg *}

Lehrstuhl für Biotechnologie, RWTH Aachen University, Worringer Weg 1, Aachen, D-52074, Germany; E-Mail: j.tenne@biotec.rwth-aachen.de

* Author to whom correspondence should be addressed; E-Mail: u.schwaneberg@biotec.rwth-aachen.de; Tel.: +49-241-80-24176; Fax: +49-241-80-223876.

Received: 6 January 2012; in revised form: 9 February 2012 / Accepted: 13 February 2012 /

Published: 22 February 2012

\begin{abstract}
Circular dichroism (CD) and deconvolution were used to study the structural integrity of a "plugged" and an "open" FhuA transmembrane channel protein in the presence of varied concentrations of tetrahydrofuran (THF), ethanol (EtOH) and chloroform/methanol (C/M). FhuA is an Escherichia coli outer membrane protein $(78.9 \mathrm{kDa})$ consisting of $22 \beta$-sheets and an internal globular cork domain which acts as an iron transporter. FhuA and the deletion variant FhuA $\Delta 1-159$ showed comparable and remarkable resistance in the presence of THF ( $\leq 40 \mathrm{vol} \%)$ and $\mathrm{EtOH}(\leq 10 \mathrm{vol} \%)$. In C/M, significant differences in structural resistance were observed (FhuA stable $\leq 10 \mathrm{vol} \%$; FhuA $\Delta 1-159 \leq 1$ vol\%). Deconvolution of CD-spectra for FhuA and FhuA $\Delta 1-159$ yielded $\beta$-sheet contents of $61 \%$ (FhuA) and 58\% (FhuA $\Delta 1-159$ ). Interestingly, FhuA and FhuA $\Delta 1-159$ had comparable $\beta$-sheet contents in the presence and absence of all three organic cosolvents. Additionally, precipitated FhuA and FhuA $\Delta 1-159$ (in $40 \mathrm{vol} \% \mathrm{C} / \mathrm{M}$ or 65 vol\% THF) redissolved by supplementing the detergent $n$-octyl-oligo-oxyethylene (oPOE).
\end{abstract}

Keywords: secondary structure; circular dichroism; membrane protein; organic cosolvent; $\beta$-barrel; FhuA; precipitation; structural integrity

Abbreviations: CD, circular dichroism; THF, tetrahydrofuran; EtOH, ethanol; C/M, chloroform/ methanol; FhuA protein, ferric hydroxamate uptake protein component A; FhuA $\Delta 1-159$, FhuA deletion variant; oPOE, n-octyl-oligo-oxyethylene; BCA-assay, bicinchoninic acid assay; IPTG, 
isopropyl- $\beta$-D-thiogalactopyranoside; $\mathrm{P} i$, potassium phosphate; EDTA, ethylenediaminetetraacetic acid; MWCO, molecular weight cut off; SDS, sodiumdodecylsulfate; $\alpha$, alpha-helical structure; $\beta$, beta-sheet structure; r, random-coil structure; FhuA WT, FhuA wildtype; O-atom, oxygen-atom; H-bond, hydrogen-bond

\section{Introduction}

Forces controlling the overall structure of proteins include electrostatic interactions, van der Waals forces, disulfide bridges and $\pi$ - $\pi$ stacking of aromatic site chains [1-3]. Membrane proteins have three regions that interact with the surrounding environment: (i) regions interacting with water; (ii) regions in direct contact with the lipid membrane; and (iii) regions facing the protein interior [2]. Membrane proteins fulfill a variety of complex functions as diffusion pores, substrate specific transporters, membrane anchors, signal transduction and compound conversion [4]. Antiparallel $\beta$-sheets of integral membrane proteins often show a meander topology, which can form a $\beta$-barrel structure and enable translocation of molecules through biological membranes. Hydrogen bonds are the main forces holding $\beta$-sheets together in a $\beta$-barrel protein. A common feature of $\beta$-barrel proteins is their frequentlydiscovered high resistance to heat, chaotropic salts, detergents and proteolysis [5]. The latter properties make $\beta$-barrel proteins attractive for applications in biocatalysis (hybrid catalysts), biotechnology (selective product recovery [6,7]), as well as medical applications through triggered compound release (e.g., $\mathrm{pH}[8]$, reduction [9] and light trigger [10]).

Gaining first insights on cosolvent effects that govern the structural integrity and solubility of $\beta$-barrel proteins are therefore important prerequisites for developing successfully hybrid catalysts, selective membranes and triggerable release systems. A "systematic" study for examining the structural integrity of an integral $\beta$-barrel membrane protein in the presence of different concentrations of organic solvents by $\mathrm{CD}^{1}$ has not, to our best knowledge, been reported.

In the current report, the organic cosolvents tetrahydrofuran (THF) [11], ethanol (EtOH) [8] and chloroform/methanol $(\mathrm{C} / \mathrm{M})$ were selected to investigate their effects on the iron transporter FhuA (ferric hydroxamate uptake protein component A) [12]. THF and EtOH were used for developing FhuA-based triggered release systems [10,13], modulating compound fluxes [14] and in hybrid catalysts synthesis [15]. FhuA is a monomeric two-domain outer membrane protein of $E$. coli with a molecular weight of $78.9 \mathrm{kDa}$ consisting of 22 antiparallel $\beta$-strands ( $C$-terminal, residues 161 to 723 ) and a $\mathrm{NH}_{2}$-terminal cork domain (residues 1 to 160) [12,16]. FhuA has a height of $69 \AA$ and an elliptical cross section of 39 to $46 \AA$ [16]. FhuA transports iron in the form of siderophores into E. coli. Furthermore it serves as a receptor for a number of bacteriophages (T5, T1, Ф80) and antibiotics (colicin M, albomycin) [17]. Removal of the cork domain (FhuA $\Delta 1-159$ ) resulted in a passive diffusion channel [12-15].

Differential scanning calorimetry (DSC) [18,19], CD [20], FTIR and NMR [21] can be used for studying the structural integrity of proteins. FTIR was excluded due to a high signal-noise ratio of FhuA and its environment, composed of lipids and detergent. The size of FhuA ( $>60 \mathrm{kDa})$, possible multimer formation and the presence of large molar excess of detergent suggested that NMR should be excluded. DSC and CD were promising choices and CD finally selected. A DSC study on thermal resistance of FhuA wildtype, showing two transition centers, due to the presence of the cork domain, 
and FhuA $\Delta 28-159$, showing one transition centre, was published [5] (FhuA: $T_{\mathrm{m} 1}=64.0{ }^{\circ} \mathrm{C}$ and $T_{\mathrm{m} 2}$ of $74.4{ }^{\circ} \mathrm{C}$, FhuA $\Delta 28-159: T_{\mathrm{m}}=61.6{ }^{\circ} \mathrm{C}$ ).

In essence, we report a $\mathrm{CD}$-based study on the structural integrity of FhuA and its mutant FhuA $\Delta 1-159$ in different concentrations of the organic solvents THF, EtOH and C/M.

\section{Results and Discussion}

In Section 2.1, the CD, as well as the deconvolution method, are described, including control experiments, in order to ensure that structural effects of cosolvents on FhuA and FhuA $\Delta 1-159$ can be determined by CD-spectra analysis. In Section 2.2, the obtained resistance results of FhuA and FhuA $\Delta$ 1-159 in varied concentrations of THF, $\mathrm{EtOH}$ and $\mathrm{C} / \mathrm{M}$ mixtures are reported and analyzed from a structural point of view. In Section 2.3, molecular reasons like water-stripping effect, dielectric constant and empirical $\log \mathrm{P}$ concept are discussed for the cosolvents THF, EtOH and C/M.

In order to simplify the discussion, FhuA or FhuA $\Delta 1-159$, in the presence of oPOE detergent (1.05 vol\%) in phosphate buffer (100 mM Pi, pH 8, 1 mM EDTA), will be referred to as "standard" buffer in contrast to cosolvent supplemented buffers.

\subsection{Results on CD-Measurements}

Characteristic CD-spectra for a $\beta$-sheet peptide have a minimum of around $215 \mathrm{~nm}$ and a maximum around $195 \mathrm{~nm}$. Peak variations are due to contributions of $\beta$-turns or antiparallel interactions between the different $\beta$-sheets [22]. Figure 1 shows the original and fitted CD-spectra of FhuA (I; upper three CD-spectra experiments) and FhuA $\Delta 1-159$ (II; lower three CD-spectra experiments) in "standard" buffer and in the presence of varied concentrations of the organic cosolvents THF (I.a/II.a), EtOH (I.b/II.b) and C/M (I.c/II.c). The CD-spectra of FhuA and FhuA $\Delta 1-159$ in a "standard" buffer show that FhuA and FhuA $\Delta 1-159$ are composed of a $\beta$-sheet structure. The recorded CD-spectra are nearly identical to a first recorded CD-spectrum of FhuA wildtype in $10 \mathrm{mM}$ Tris (pH 7.2), $10 \mathrm{mM} \mathrm{NaCl}$ and $16.5 \mathrm{mM}$ octyl glucoside buffer [23]. Comparison of FhuA to FhuA $\Delta 1-159$ in THF and EtOH show a similar trend and high resistance of the $\beta$-barrel structure towards the organic cosolvents. In both cases the maximum at around $195 \mathrm{~nm}$ decreases significantly in the presence of $40 \mathrm{vol} \%$ organic cosolvent. The single minimum at around $215 \mathrm{~nm}$, which is typical for $\beta$-sheets, is decreased step-wise by the addition of cosolvent to the wildtype FhuA protein. In contrast to FhuA, addition of small amounts of THF or EtOH to FhuA $\Delta 1-159$ directly lowers the minima but the overall shape remains almost the same during the addition of any concentration of cosolvents. However, one has to be careful not to overinterpret differences in CD-spectra, for instance in the range of 205-240 nm (see Figure 1 IIa). As a general trend it was found that the resistance of the secondary structure of FhuA proteins towards $\mathrm{C} / \mathrm{M}$ is much lower, compared to THF or EtOH (see Figure 1). In addition, the secondary structure of FhuA $\Delta 1-159$ is less resistant towards THF, EtOH and C/M, compared to the wildtype FhuA. 
Figure 1. The respective amount of organic cosolvent (THF, EtOH or C/M) was added to $180 \mathrm{ng} / \mu \mathrm{L}$ of FhuA or FhuA $\Delta 1-159$ in a total volume of $150 \mu \mathrm{L}$. Samples were stirred for 75 min. CD-spectra were recorded using the Olis spectrapolarimeter model SDM 17, within a SUPRASIL cuvette with a pathlength of $0.5 \mathrm{~mm}$. After subtraction of buffer baseline spectra and smoothing using a Savitzky-Golay smoothing filter, results were expressed in milli degrees. Data were converted into mean residue ellipticity and fitted using the CONTIN algorithm, implemented in the Dichroprot software. Original data (dotted lines) and fitted data (solid lines) of (I) FhuA and (II) FhuA $\Delta 1-159$ in (a) THF; (b) $\mathrm{EtOH}$ and (c) $\mathrm{C} / \mathrm{M}$ are shown. Samples marked with one asterisk showed precipitation of FhuA or FhuA $\Delta$ 1-159 upon addition of organic cosolvent which redissolved after incubation. Two asterisks indicate the FhuA or FhuA $\Delta 1-159$ precipitation events in which FhuA or FhuA $\Delta$ 1-159 did not fully redissolve during CD-measurements.
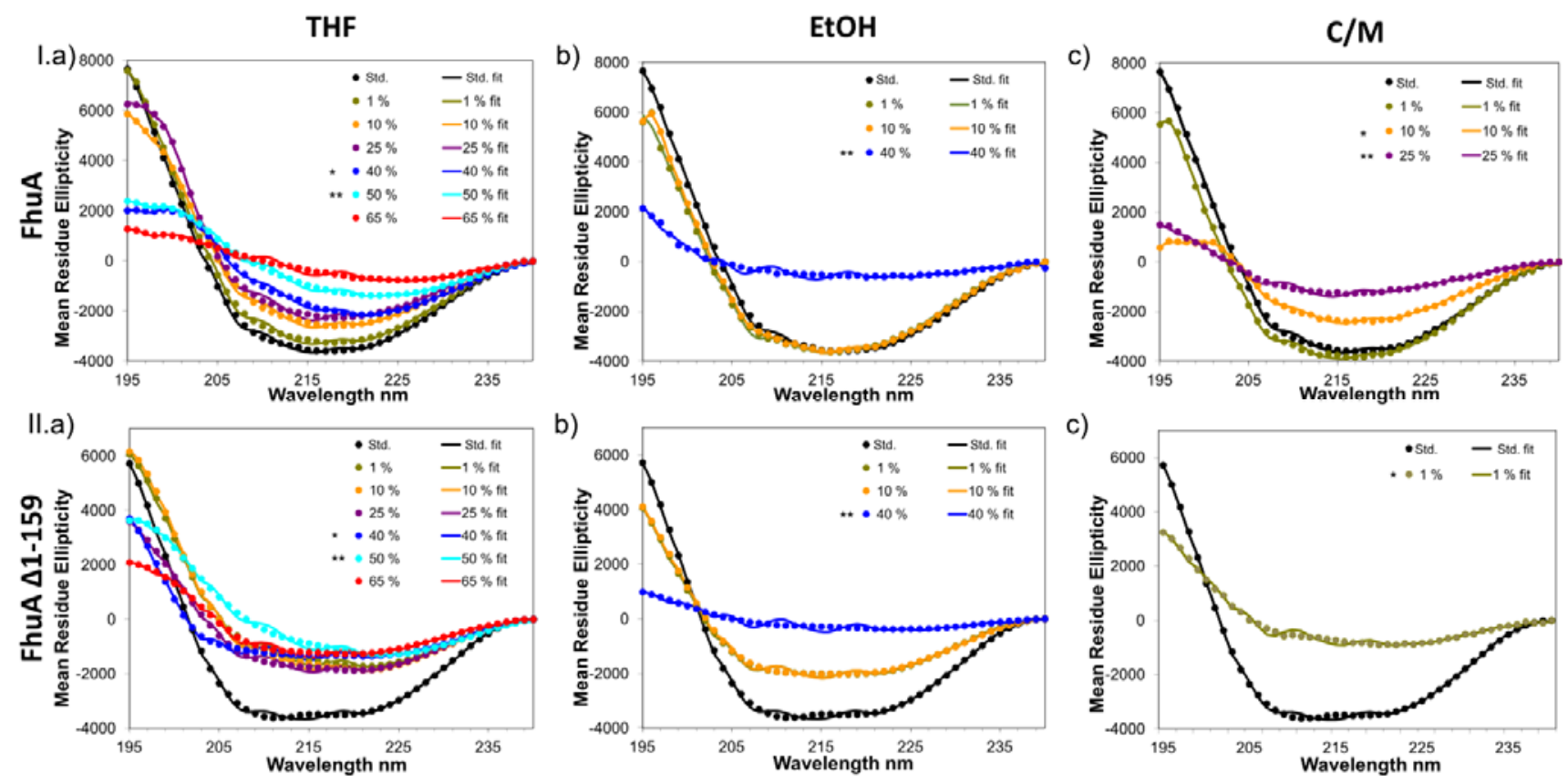

Supplementation of $40 \mathrm{vol} \%$ THF or $25 \mathrm{vol} \% \mathrm{EtOH}$ (data not shown) during sample preparation of FhuA or FhuA $\Delta 1-159$ turned the protein solution from transparent to turbid. This phenomenon disappeared after incubation (75 min; see Figure 1, *). In the case of C/M, $10 \mathrm{vol} \% \mathrm{C} / \mathrm{M}$ (FhuA) or 1 vol\% (FhuA $\Delta 1-159$ ) resulted in similar effects. Cosolvent addition causing turbidity may be attributed to a local overconcentration of the cosolvent reducing FhuA and FhuA $\Delta 1-159$ solubility leading to precipitation of FhuA or FhuA $\Delta 1-159$. Supporting Material, Figure C, illustrates the precipitation and dissolution process and Fig. D (SDS-gel of precipitate) shows FhuA $\Delta 1-159$ which was precipitated. Precipitated FhuA $\Delta 1-159$ could be redissolved by supplementing a buffer solution containing oPOE (3 vol\%) or urea (4 M) and incubating ( $2 \mathrm{~h}$, see Supplementary Material, Table E). The latter indicates that the detergent oPOE plays a pivotal for the water solubility of FhuA or FhuA $\Delta 1-159$ in the presence of cosolvents (THF, EtOH, C/M).

Figure 1 shows the measured (dotted line) and fitted (solid line; CONTIN algorithm) CD-data. Recorded and fitted data are in agreement showing, for instance, a very similar shape, which is a good hint that the CONTIN algorithm can be used to analyze FhuA or FhuA $\Delta 1-159$ and likely other 
$\beta$-barrel shaped outer membrane proteins which are rich in $\beta$-sheet content. On the basis of the fitted data, the CONTIN algorithm "deconvolutes" the data, displaying the structural elements alpha-helix $(\alpha)$, beta-sheet $(\beta)$ and random coil (r) of the respective samples (see Table 1).

Table 1. CD-spectra of FhuA and FhuA $\Delta 1-159$ in the presence of varied amounts of the organic cosolvents $\mathrm{THF}$, EtOH or $\mathrm{C} / \mathrm{M}$. CD-spectra were recorded in milli degrees and converted into mean residue ellipticity and fitted with the CONTIN algorithm implemented in the Dichroprot software, determining the amount of $\alpha$-helix, $\beta$-sheet and random coil content of the respective samples.

\begin{tabular}{ccccccc}
\hline & \multicolumn{6}{c}{ Secondary structure in \%-age } \\
\cline { 2 - 7 } Sample & \multicolumn{5}{c}{ FhuA WT } & \multicolumn{3}{c}{ FhuA $\mathbf{\Delta 1 - 1 5 9}$} \\
\cline { 2 - 7 } & Helix & Sheet & Random coil & Helix & Sheet & Random coil \\
\hline Standard & 4 & 61 & 34 & 3 & 58 & 39 \\
1 vol\% THF & 3 & 64 & 33 & 3 & 64 & 33 \\
10 vol\% THF & 1 & 66 & 33 & 1 & 66 & 33 \\
25 vol\% THF & 0 & 73 & 26 & 0 & 73 & 26 \\
40 vol\% THF & 0 & 60 & 40 & 0 & 60 & 40 \\
50 vol\% THF & 0 & 65 & 35 & 0 & 65 & 35 \\
65 vol\% THF & 0 & 67 & 33 & 0 & 67 & 33 \\
1 vol\% EtOH & 6 & 63 & 31 & 0 & 63 & 37 \\
10 vol\% EtOH & 8 & 73 & 19 & 0 & 63 & 37 \\
40 vol\% EtOH & 0 & 68 & 32 & 0 & 70 & 30 \\
1 vol\% C/M & 8 & 66 & 26 & 0 & 69 & 31 \\
10 vol\% C/M & 3 & 62 & 35 & 0 & 68 & 32 \\
25 vol\% C/M & 0 & 67 & 33 & 0 & 67 & 33 \\
\hline
\end{tabular}

Deconvolution of CD-data shows almost no changes in the amounts of each structural element; $\alpha$-helix, $\beta$-sheet or random coil, upon addition of any concentration of organic cosolvent. We verified these results by repeating the experiments three times with different FhuA or FhuA $\Delta 1-159$ preparation. Interestingly, even in the presence of high concentrations of organic cosolvents, partial amounts of FhuA and FhuA $\Delta$ 1-159 remained in solution and yielded CD-spectra which indicate that the overall $\beta$-barrel structure is still intact.

Reduced intensities of the FhuA or FhuA $\Delta 1-159$ structure from a distinctive $\beta$-sheet structure (Figure 1) could mainly be based on the aggregation of FhuA proteins in the presence of organic cosolvents and can be considered as a three-stage phenomenon: (1) The protein structure is very stable, only slight alterations of the original spectra can be seen; (2) Addition of a certain amount of organic cosolvent leads to aggregation of the protein (appearance of cloudiness) while after an incubation time of 75 minutes, FhuA dissolves again, forming a transparent solution. CD-spectra still show the typical minimum and maximum of a $\beta$-sheet peptide, but are less intense, compared to stage (1). It is assumed that in stage (2), the local concentration of organic cosolvent leads to partial protein aggregation. In stage (3), the typical $\beta$-sheet CD-spectra of FhuA or FhuA $\Delta 1-159$ is reduced in absolute values but retains its overall shape. The latter could be explained by the assumption that majority of the FhuA or FhuA 
$\Delta 1-159$ precipitate while a small fraction remains in solution despite of the presence of the organic cosolvent (see Supplementary Material, Figures C and D).

In the following, the three stage phenomenon will be explained on the basis of FhuA and FhuA $\Delta$ 1-159 in the presence of THF (Figure 1 I.a/II.a). FhuA protein variants show structural integrity (stage 1) up to $25 \mathrm{vol} \%$ THF. The spectra exhibit the typical structure of a protein which is rich in $\beta$-sheet content. 40 vol\% THF leads to protein aggregation, however FhuA proteins redissolve during an incubation period (75 min; stage 2). The addition of $50 \mathrm{vol} \%$ or $65 \mathrm{vol} \%$ THF leads to irreversible precipitation of FhuA or FhuA $\Delta 1-159$ during the $75 \mathrm{~min}$ incubation period (stage 3). Precipitate could be redissolved in a detergent (oPOE; 3 vol\%) solution (see Supplementary Material, Figure E). Interestingly, CD-spectra still showed a $\beta$-structure which is based on the fact that not all FhuA or FhuA $\Delta$ 1-159 molecules aggregated in presence of THF (see deconvolution of CD-spectra, Table 1).

\subsection{Secondary Structure Analysis}

In order to determine the percentage of each structural element from a CD-spectrum, it is important to employ the right method for analysis. This is especially important for the analysis of membrane proteins in which for instance the origin and the number of spectra in the reference data sets are decisive for deconvolution [24]. For secondary structure analysis of FhuA, the methods, with their respective percentage of $\beta$-sheets, are listed below: Secondary structure assignment of FhuA wildtype (FhuA WT, PDB entry 2FCP) reveals about 49\% $\beta$-sheet content [12], while DSSP [25] indicates 53\% $\beta$-sheets. Deconvolution of FhuA WT CD-data by the Andrade algorithm yields a $\beta$-sheet content of $51 \%$ [23]. It is commonly known that determination of $\beta$-sheet content in $\beta$-barrel proteins varies for the same $\beta$-barrel protein, even if the same deconvolution algorithms are employed. For instance, a report on the deletion variant FhuA $\Delta 1-159$ analyzed with CONTIN [26] shows a $\beta$-sheet content in the range of $60-65 \%$ [27]. The latter $\beta$-sheet contents are in reasonably good agreement with our $\beta$-sheet content values in Table 1 (58-73\%).

Variations in $\beta$-sheet contents depend often on sample preparation and protein "purity". For instance, $\beta$-sheets contribute four times less to $\mathrm{CD}$-absorbance measurements than $\alpha$-helical structure, so that even small amounts of impurities can affect $\beta$-sheet content values [28]. In addition, $\beta$-strands have in general a higher structural flexibility (barrels, sheets, propellers, $\beta$-helices) [22].

$\mathrm{CD}$ was originally developed for water-soluble proteins. Therefore, analysis of highly hydrophobic $\beta$-barrel proteins with a detergent shell is challenging for the CD-method. Studies on FhuA [27] and OmpA (outer membrane protein A) [29] illustrated that depending on the detergent-like environment and its concentration around the protein (in this case either defined by polymer, $n$-octyl-polyoxyethylene or different lipids), wavelength shifts can occur (e.g., blue shift in presence of polymer [27]). In addition, Chen and Wallace (1997) reported that non-aqueous solvents shift spectral peak positions in CD-spectra. Depending on the nature of the solvent, shifts can be either red-shifts (apolar solvents) or blue-shifts (polar solvents) [24]. The shift of the maxima at round $195 \mathrm{~nm}$ could be caused by the cosolvent effect reported by Chen and Wallace (1997).

CD-data analysis gives good clues on whether FhuA or FhuA $\Delta 1-159$ are correctly folded and stable in the presence of cosolvents besides maxima shifts caused by cosolvents, detergent effects on 
CD-spectra and general challenges such as selection of a suitable deconvolution algorithm and high purity of FhuA or FhuA $\Delta 1-159$.

FhuA is more stable than the cork-domain lacking variant FhuA $\Delta 1-159$. We assume that this is the result of more than 60 hydrogen bonds and nine salt bridges between the protein interior and the cork domain [16]. Bonhivers et al. (2001) performed stability studies of FhuA and FhuA $\Delta 021-128$, the latter one lacking most parts of the cork domain, and concluded that the wildtype is thermally more stable (FhuA: $T_{\mathrm{m} 1}=64{ }^{\circ} \mathrm{C}$ (cork domain unfolding) and $T_{\mathrm{m} 2}=74.4{ }^{\circ} \mathrm{C}$ (barrel unfolding)), more resistant to trypsin proteolysis and more stable to denaturing agents. These trends correspond well to our findings in respect of higher organic cosolvent resistance of the wildtype FhuA.

\subsection{Solvent Effects}

The structural integrity of proteins is determined by interactions within the protein and its environment. Protein molecules in solution are surrounded by a hydration shell in which water molecules are mainly attached to the protein surface by hydrogen bonds. Structural loss or denaturation of proteins by organic cosolvents is based on disturbance of this hydration shell [30] or disruption of the hydrophobic core.

Due to their high affinity to water, hydrophilic solvents will most likely strip the water from the protein. The hydrophilicity is directly proportional to the dielectric constant $\varepsilon$ and the donor/acceptor characteristics of the solvent. In some cases, the water-protein interactions are so tight that hydrophilic solvents have difficulties in striping water away [31]. $\mathrm{MeOH}$ and $\mathrm{EtOH}(\varepsilon=33$ and 30) are able to accept and to donate hydrogen bonds to water. They can harm the hydrophilic parts of proteins. THF $(\varepsilon=7.5)$ is exclusively an H-bond acceptor while $\mathrm{CHCl}_{3}(\varepsilon=4.8)$ is neither an acceptor nor a donor.

FhuA wildtype and its deletion mutant FhuA $\Delta 1-159$ are integral membrane proteins and therefore the hydrophilic parts of the protein are located in the upper and lower rim of the $\beta$-barrels FhuA or FhuA $\Delta 1-159$ and contribute to the solubility in water.

The middle section of the $\beta$-barrel itself is highly hydrophobic and covered with detergent. In nature, the FhuA membrane-embedded surface consists of $45.4 \%$ hydrophobic residues [4]. Therefore it was assumed that solvents with a high $\log \mathrm{P}$ value $\left(\mathrm{CHCl}_{3}=2.0\right.$, THF $=0.53$, EtOH $=-0.24$, $\mathrm{MeOH}=-0.76$ ) are less harmful for hydrophobic membrane proteins. For FhuA or FhuA $\Delta 1-159$ the expectation turned out to be valid for $\mathrm{THF}$ and $\mathrm{EtOH}$. A C/M mixture should, due to its dielectric constants and the $\log \mathrm{P}$ values of each component, affect FhuA or FhuA $\Delta 1-159$ in a comparable manner to THF or EtOH. However, why does the C/M mixture directly lead to protein aggregation? In the case of FhuA, the hydrophobic part of the purified FhuA or FhuA $\Delta 1-159$ is surrounded by the detergent oPOE which assists in FhuA or FhuA $\Delta$ 1-159 solubility. In subsequent control experiments we investigated whether oPOE solubility depends on cosolvent concentrations. THF and EtOH can be mixed with 3 vol\% oPOE without showing changes in the sample turbidity (see Supplementary Material, Table F and Figure $\mathrm{G}$ ). The addition of $\mathrm{C} / \mathrm{M}$ to 3 vol\% oPOE caused an immediate change from a clear solution to a cloudy one that became clear over time. However, in most cases, "lipid-like" droplets remained visible after incubation. In conclusion, we do not expect that the organic solvent mixture $\mathrm{C} / \mathrm{M}$ itself leads to protein aggregation/precipitation. Rather, FhuA or FhuA $\Delta 1-159$ 
precipitation is governed by interactions between $\mathrm{C} / \mathrm{M}$ and oPOE which reduces FhuA or FhuA $\Delta 1-159$ solubility.

In the subsequent paragraph, interactions of THF, EtOH, and $\mathrm{C} / \mathrm{M}$ on FhuA or FhuA $\Delta 1-159$ will be discussed on a molecular level. THF leads to H-bond formations between THF and water, forming a water shell around the THF molecules. As more THF is supplemented, competition of THF $O$-atoms for H-bond formation increases [32]. Our study suggests that this leads to disruption of bulk water of FhuA or FhuA $\Delta 1-159$, causing a minor alteration of the secondary structure (Figure 1). The water interacting with FhuA or FhuA $\Delta 1-159$ is not disrupted because most THF gets "caged" by water. It is known that until $0.2-0.4$ mole fraction (53-73\%), $O$-atoms of THF are not competing efficiently as hydrogen bond acceptors compared to water, preferring THF-THF interactions [33]. Increasing the THF (0.4-0.7 mole fraction; 73-90\%) would most probably cause complete replacement of $\mathrm{H}$-bonds between water molecules by H-bonds between THF molecules [32], leading to competition between the water surrounding the FhuA or FhuA $\Delta 1-159$.

Small amounts of EtOH show more perturbation of the protein structure in comparison to THF. Water molecules form a distorted cage around the EtOH molecule (located mainly around the methyl group) [34]. Higher EtOH concentrations perturb the FhuA or FhuA $\Delta 1-159$ water because of hydrogen bond competition with the hydroxyl group of EtOH and van der Waals interactions with the alkyl chain. Perturbation of the water comes with rearrangement of the detergent, covering FhuA or FhuA $\Delta 1-159$. The latter might result in decreased solubility and aggregation of FhuA or FhuA $\Delta 1-159$.

FhuA WT and FhuA $\Delta 1-159$ were both least stable in mixtures of $\mathrm{C} / \mathrm{M}$. Chloroform cannot act as a hydrogen bond acceptor and because of the chloride's van der Waals dimension, hydrogen-bond donor characteristics are very limited. It is highly likely that the reasons for FhuA or FhuA $\Delta 1-159$ aggregation lie in the interaction of the organic cosolvents with the detergent oPOE (see Supplementary Material, Table F and Figure G) which covers the hydrophobic middle part of FhuA or FhuA $\Delta 1-159$. As a result $\mathrm{MeOH}$ might, due to the interactions between $\mathrm{CHCl}_{3}$ and oPOE, obtain easier access to water molecules and the H-bond network of FhuA or FhuA $\Delta 1-159$, which might promote FhuA or FhuA $\Delta 1-159$ aggregation and finally precipitation.

\section{Experimental Section}

All chemicals were of analytical grade quality or higher, purchased from Applichem (Darmstadt, Germany) and Sigma-Aldrich Chemie (Taufkirchen, Germany). The detergent $n$-octyl-oligo-oxyethylene (oPOE) was purchased from Enzo Life Sciences (Lörrach, Germany). Protein concentrations were determined using the bicinchoninic acid assay (BCA-assay) from Pierce Chemical Co (Rockford, USA). The organic cosolvents were acquired from Sigma-Aldrich (THF, M) and Applichem (EtOH, C). All experiments were carried out using Eppendorf $\operatorname{Research}^{\circledR}(0.5-10 \mu \mathrm{L}, 10-100 \mu \mathrm{L}, 100-1000 \mu \mathrm{L})$ pipettes (Hamburg, Germany) with tips (S1111-4000, S1112-1020-c, S1111-0006-c) from Star Lab (Ahrensburg, Germany).

\subsection{Strains}

FhuA was provided on the pHK 763 vector by Prof. Volkmar Braun [35] and cloned into pPR-IBA1 (isopropyl- $\beta$-D-thiogalactopyranoside (IPTG) induced), using an EcoRI restriction site at the 5'-end 
and an XhoI restriction site at the 3'-end. FhuA and the FhuA $\Delta 1-159$ deletion variant contain as previously described a signal sequence to the outer E. coli membrane [36].

\subsection{Expression and Extraction of FhuA and FhuA $\Delta 1-159$}

The FhuA plasmids were transformed into the expression host E. coli $\mathrm{B}^{\mathrm{E}}$ strain BL 21 (DE3) omp8 $\left(\mathrm{F}^{-} h s d S_{B}\left(\mathrm{r}_{\mathrm{B}}-\mathrm{m}_{\mathrm{B}}-\right)\right.$ gal ompT dcm (DE3) $\Delta l a m B$ ompF ::Tn5 $\Delta$ ompA $\Delta$ ompC) [37] and expressed in the Biostat ED fermenter from Sartorius (Göttingen, Germany), with a working volume of $10 \mathrm{~L}$ (tryptone-yeast extract media ( $1 \%$ tryptone, $0.5 \%$ yeast extract, $0.5 \% \mathrm{NaCl}), 37^{\circ} \mathrm{C}, 150 \mathrm{rpm}$, six-flate disc-turbine, sparged with air). The protocol for expression and extraction of the FhuA variants was carried out as described by Nallani et al. [36], with slight modifications: inoculation volume was calculated to reach $\mathrm{OD}_{600}$ of 0.1 in the main culture. After induction with IPTG, cells had grown further at $37{ }^{\circ} \mathrm{C}$ until $\mathrm{OD}_{600}$ reached 2.5. The culture broth was harvested by centrifuging (3220 g, $20 \mathrm{~min}, 4^{\circ} \mathrm{C}$, Eppendorf 5810 D, Hamburg, Germany). Pellets were kept at $-20{ }^{\circ} \mathrm{C}$ until proteins were extracted. All buffers are potassium phosphate $(\mathrm{P} i)$ based $(100 \mathrm{mM})$. After resuspension in lysis buffer (100 mM Pi, pH 8, $5 \mathrm{mM} \mathrm{MgCl}_{2}, 0.1 \mathrm{mM} \mathrm{CaCl}_{2}$ ), cells were disrupted by a high-pressure homogenizer $(3 \times 1800$ bar pressure, Emulsifelx-C3 Homogenizer, Avestin, Ottawa, Canada). For incubation, an extraction buffer (100 mM Pi, pH 8, $5 \mathrm{mM} \mathrm{MgCl}_{2}, 0.1 \mathrm{mM} \mathrm{CaCl}_{2}, 2 \%$ Triton-X 100) was supplemented. Outer membrane fractions were isolated by centrifuging $\left(38,500 \mathrm{~g}, 45 \mathrm{~min}, 4{ }^{\circ} \mathrm{C}\right.$, Thermo Scientific, Sorvall RC-6 Plus, Rotor F-21S-8X50Y, Hamburg, Germany) and washed three times with $\mathrm{P} i$-buffer (100 mM, pH 8.0). The two subsequent ultracentrifuge steps were performed in a Beckmann Coulter Optima TM L-60 Ultracentrifuge, rotor 60-Ti (170,000 g), using $100 \mathrm{mM} \mathrm{Pi,pH} \mathrm{8,}$ $1 \mathrm{mM}$ EDTA and either 0.1 vol $\%$ or 3 vol $\%$ oPOE.

\subsection{Purification and Concentrating of FhuA and FhuA $\Delta 1-159$}

Protein impurities were removed by gel-filtration (Sephadex G25-fine, GE Healthcare, München, Germany). FhuA $\Delta 1-159$ was concentrated (1800 g, $40 \mathrm{~min}, 4{ }^{\circ} \mathrm{C}$, Eppendorf $5810 \mathrm{R}$, Hamburg, Germany) using a 10 kDa MWCO filter unit (Vivaspin 15, Sartorius, Göttingen, Germany).

\subsection{Determination of Protein Purity and Concentration}

Protein purity was determined by $10 \%$ acrylamide gels with $0.1 \%$ SDS running in a Biorad Mini-PROTEAN system (Hercules, CA, USA). For visualization of the bands, coomassie staining was used [38]. Protein concentrations were determined by the BCA-assay (Thermo Scientific, Rockford, USA). The BCA-assay quantifies the total protein amount; the IMAGE-J (Image Processing and Analysis in Java, Version 1.41) program was used to determine the amount of purified FhuA $(1300 \mathrm{ng} / \mu \mathrm{L})$ and FhuA $\Delta 1-159(600 \mathrm{ng} / \mu \mathrm{L})$. Figure $\mathrm{H}$ in the Supporting Material shows the purified FhuA and FhuA $\Delta 1-159$ used in CD measurements.

\subsection{Sample Preparation for CD Spectroscopy}

The total sample volume was $150 \mu \mathrm{L}$ in each measurement. $180 \mathrm{ng} / \mu \mathrm{L}$ FhuA (leading to $20.7 \mu \mathrm{L}$ ) or FhuA $\Delta 1-159$ (leading to $45 \mu \mathrm{L}$ ) were pipetted into a small glass tube (S 4-W, transparent, $\mathrm{Nr}$. 
300095-100, $4 \mathrm{~mL}, 44.5 \times 14.5 \mathrm{~mm}$, Chromatographie Service GmbH, Langerwehe, Germany), standing on a magnetic stirrer (stirring magnet: Nr. 001.106, $6 \times 3 \mathrm{~mm}$, Cowie, Middlesbrough, United Kingdom, stirring unit: Mot 2.5, Ika Werke RT10, Ika Werke, Staufen, Germany) with either $31.8 \mu \mathrm{L}$ or $7.5 \mu \mathrm{L}$ oPOE-based buffer (100 mM Pi, pH 8, $1 \mathrm{mM}$ EDTA, 3 vol\% oPOE). The protein in the oPOE-buffer always displayed $35 \mathrm{vol} \%$ of the total sample solution. The remaining $65 \mathrm{vol} \%$ of the sample were prepared by the respective amounts of buffer (100 mM Pi, pH 8, $1 \mathrm{mM}$ EDTA) and organic solvents (THF, EtOH or C/M (1:1 v/v), 0\%, 1\%, 10\%, 25\%, 40\%, 50\%, 65\% (v/v). Both the buffer and the organic solvent were slowly added by pipetting to the protein sample. Stirring was carried out for $75 \mathrm{~min}$ (in the subsequent text termed as incubation time). Each sample (150 $\mu \mathrm{L})$ was carefully transferred with an Eppendorf pipette onto a Hellma ${ }^{\circledR}$ SUPRASIL cuvette (Hellma GmbH \& Co. KG, Müllheim, Germany) with a pathlength of $0.5 \mathrm{~mm}$. Sample analysis was performed at room temperature with Olis SDM 17 CD (Olis, Bogart, USA).

\subsection{Secondary Structure Determination of FhuA Variants by CD Spectroscopy}

For each sample, five scans from 195 to $240 \mathrm{~nm}$ were recorded and averaged. $195 \mathrm{~nm}$ was selected as a benchmarking wavelength since organic solvents (like THF) have an increased absorbance below $195 \mathrm{~nm}$ leading to high noise/signal ratios. The latter interferes with deconvolution of recorded CD-spectra. The bandwidth was adjusted to $2 \mathrm{~nm}$, the step width to $1 \mathrm{~nm}$. As baseline, buffer (100 $\mathrm{mM}$ $\mathrm{P} i, \mathrm{pH}$ 8, $1 \mathrm{mM}$ EDTA, 1.05 vol\% oPOE) without FhuA was employed in all experiments since the baseline in presence of cosolvent did not deviate significantly from the baseline of the buffer without cosolvent (see Supplementary Material, Figure A; example THF). In addition, deconvolution confirmed that the THF-baseline or oPOE-baseline can be used as reference for analyzing CD since both yield comparable $\beta$-sheet contents (see Supplementary Material, Table B). CD-spectra were smoothed by the Savitzky-Golay filter (Olis Global Works software package), and results were given as milli degrees and converted into mean residue ellipticity. To quantify the extent of changes in the secondary structure of FhuA and FhuA $\Delta 1-159$, the CONTIN algorithm [26], originally developed by Provencher and Glockner [39], was used. The algorithm is implemented in the Dichroprot software [40] and includes a spectra database of known secondary peptide-structures in order to predict the content of $\alpha$-helix, $\beta$-sheet and random coil in the respective sample.

\section{Conclusions}

FhuA and FhuA $\Delta 1-159$ showed a structural integrity in up to $40 \mathrm{vol} \% \mathrm{THF}$ and $10 \mathrm{vol} \% \mathrm{EtOH}$. In $\mathrm{C} / \mathrm{M}$ mixtures, FhuA aggregates, starting from $10 \mathrm{vol} \%$ of organic cosolvent, while the deletion variant already indicates strong aggregation above $1 \mathrm{vol} \% \mathrm{C} / \mathrm{M}$. Independent of the structural integrity of FhuA or FhuA $\Delta 1-159$, deconvolution of CD-spectra showed that even at high concentrations of organic cosolvent, FhuA or FhuA $\Delta 1-159$ did not greatly alter the examined structure. Furthermore, the cork domain improves the resistance of FhuA in organic cosolvents.

Our study showed that it is possible to investigate structural integrity of the $\beta$-barrel protein FhuA in organic cosolvents by CD-spectra analysis. We gained first insights of cosolvent effects on stability and solubility of FhuA or FhuA $\Delta 1-159$. The developed protocol opens opportunities to further study interactions of FhuA and other $\beta$-barrel proteins in non-natural environments. Polymersomes and 
artificial membranes offer novel applications for engineered membrane channel proteins such as hosts for hybrid catalysts or as filters in membranes or medical applications.

\section{Acknowledgments}

This work was performed as part of the Cluster of Excellence "Tailor-Made Fuels from Biomass", which is funded by the Excellence Initiative by the German federal and state governments to promote science and research at German universities.

We appreciate the assistance of M. Bocola (Institute of Biotechnology, RWTH Aachen University) and acknowledge valuable discussions of M. Arlt (Institute of Biotechnology, RWTH Aachen University).

\section{References}

1. Cramer, W.; Engelman, D.; Von Heijne, G.; Rees, D. Forces involved in the assembly and stabilization of membrane proteins. FASEB J. 1992, 6, 3397-3402.

2. Haltia, T.; Freire, E. Forces and factors that contribute to the structural stability of membrane proteins. Biochim. Biophys. Acta 1995, 1241, 295-322.

3. Kelly, S.M.; Price, N.C. The use of circular dichroism in the investigation of protein structure and function. Curr. Protein Pept. Sci. 2000, 1, 349-384.

4. Koebnik, R.; Locher, K.P.; Van Gelder, P. Structure and function of bacterial outer membrane proteins: Barrels in a nutshell. Mol. Microbiol. 2000, 37, 239-253.

5. Bonhivers, M.; Desmadril, M.; Moeck, G.S.; Boulanger, P.; Colomer-Pallas, A.; Letellier, L. Stability studies of FhuA, a two-domain outer membrane protein from Escherichia coli. Biochemistry 2001, 40, 2606-2613.

6. Onaca, O.; Nallani, M.; Ihle, S.; Schenk, A.; Schwaneberg, U. Functionalized nanocompartments (Synthosomes): Limitations and prospective applications in industrial biotechnology. Biotechnol. J. 2006, 1, 795-805.

7. Mohammad, M.M.; Howard, K.R.; Movileanu, L. The redesign of a plugged beta-barrel membrane protein. J. Biol. Chem. 2010, 286, 8000-8013.

8. Ihle, S.; Onaca, O.; Rigler, P.; Hauer, B.; Rodríguez-Ropero, F.; Fioroni, M.; Schwaneberg, U. Nanocompartments with a $\mathrm{pH}$ release system based on an engineered OmpF channel protein. Soft Matter 2011, 7, 532-539.

9. Onaca, O.; Sarkar, P.; Roccatano, D.; Friedrich, T.; Hauer, B.; Grzelakowski, M.; Güven, A.; Fioroni, M.; Schwaneberg, U. Functionalized nanocompartments (synthosomes) with a reductiontriggered release system. Angew. Chem. 2008, 47, 7029-7031.

10. Güven, A.; Dworeck, T.; Fioroni, M.; Schwaneberg, U. Residue K556: A light triggerable gatekeeper to sterically control translocation in FhuA. Adv. Eng. Mater. 2011, 13, 324-329.

11. Muhammad, N.; Dworeck, T.; Fioroni, M.; Schwaneberg, U. Engineering of the E. coli outer membrane protein FhuA to overcome the hydrophobic mismatch in thick polymeric membranes. $J$. Nanobiotechnol. 2011, 9, 1-9. 
12. Ferguson, A.D.; Hofmann, E.; Coulton, J.W.; Diederichs, K.; Welte, W. Siderophore-mediated iron transport: Crystal structure of FhuA with bound lipopolysaccharide. Science 1998, 282, 2215-2220.

13. Güven, A.; Fioroni, M.; Hauer, B.; Schwaneberg, U. Molecular understanding of sterically controlled compound release through an engineered channel protein (FhuA). J. Nanobiotechnol. 2010, 8, 1-9.

14. Nallani, M.; Onaca, O.; Gera, N.; Hildenbrand, K.; Hoheisel, W.; Schwaneberg, U. A nanophosphor-based method for selective DNA recovery in Synthosomes. Biotechnol. J. 2006, $1,828-834$.

15. Tenne, S.-J.; Dworeck, T.; Güven, A.; Ihle, S.; Fioroni, M.; Schwaneberg, U. Design of Triggered Channel Proteins. In Proceedings of PEP Talk Conference, San Diego, CA, USA, 10-14 January 2011.

16. Locher, K.P.; Rees, B.; Koebnik, R.; Mitschler, A.; Moulinier, L.; Rosenbusch, J.P.; Moras, D. Transmembrane signaling across the ligand-gated FhuA receptor: Crystal structures of free and ferrichrome-bound states reveal allosteric changes. Cell 1998, 95, 771-778.

17. Schultz, G.; Ullrich, F.; Heller, K.J.; Braun, V. Export and activity of hybrid Fhua'-'lut receptor proteins and of truncated Fhua' proteins of the outer membrane of Escherichia coli. Mol. Gen. Genet. 1989, 216, 230-238.

18. Destoumieux-Garzón, D.; Duquesne, S.; Peduzzi, J.; Goulard, C.; Desmadril, M.; Letellier, L.; Rebuffat, S.; Boulanger, P. The iron-siderophore transporter FhuA is the receptor for the antimicrobial peptide microcin J25: Role of the microcin Val11-Pro16 $\beta$-hairpin region in the recognition mechanism. Biochem. J. 2005, 389, 869-876.

19. Borisover, M.D.; Zakharychev, D.V.; Solomonov, B.N. Effect of solvent composition on DSC exothermic peak of human serum albumin suspended in pyridine-n-hexane mixtures. $J$. Therm. Anal. Calorim. 1999, 55, 85-92.

20. Manavalan, P.; Johnson, W.C. Protein secondary structure from circular dichroism spectra. $J$. Biosci. 1985, 8, 141-149.

21. Pebay-Peyroula, E. Biophysical Analysis of Membrane Proteins: Investigating Structure and Function, 1st ed.; Wiley-VCH: Weinheim, Germany, 2008; pp. 55-79.

22. Wallace, B.A.; Lees, J.G.; Orry, A.J.W.; Lobley, A.; Janes, R.W. Analyses of circular dichroism spectra of membrane proteins. Protein Sci. 2003, 12, 875-884.

23. Boulanger, P.; le Maire, M.; Bonhivers, M.; Dubois, S.; Desmadril, M.; Letellier, L. Purification and structural and functional characterization of FhuA, a transporter of the Escherichia coli outer membrane. Biochemistry 1996, 35, 14216-14224.

24. Chen, Y.; Wallace, B.A. Secondary solvent effects on the circular dichroism spectra of polypeptides in non-aqueous environments: Influence of polarisation effects on the far ultraviolet spectra of alamethicin. Biophys. Chem. 1997, 65, 65-74.

25. Kabsch, W.; Sander, C. Dictionary of protein secondary structure: Pattern recognition of hydrogen-bonded and geometrical features. Biopolymers 1983, 22, 2577-2637.

26. Provencher, S. An eigenfunction expansion method for the analysis of exponential decay curves. $J$. Chem. Phys. 1976, 64, 2772-2777.

27. Dworeck, T.; Petri, A.-K.; Muhammad, N.; Fioroni, M.; Schwaneberg, U. FhuA deletion variant 
[Delta]1-159 overexpression in inclusion bodies and refolding with Polyethylene-Poly(ethylene glycol) diblock copolymer. Protein Expr. Purif. 2011, 77, 75-79.

28. Pancoska, P.; Blazek, M.; Keiderling, T.A. Relationships between secondary structure fractions for globular proteins. Neural network analyses of crystallographic data sets. Biochemistry 1992, $31,10250-10257$.

29. Kleinschmidt, J.H.; Tamm, L.K. Secondary and tertiary structure formation of the $\beta$-barrel membrane protein OmpA is synchronized and depends on membrane thickness. J. Mol. Biol. 2002, 324, 319-330.

30. Rupley, J.A.; Gratton, E.; Careri, G. Water and globular proteins. Trends Biochem. Sci. 1983, $8,18-22$.

31. Natarajan, K.R. Biocatalysis in organic solvents. J. Chem. Educ. 1991, 68, 13-16.

32. Glew, D.N.; Watts, H. Aqueous nonelectrolyte solutions. Part XII. Enthalpies of mixing of water and deuterium-oxide with tetrahydrofuran. Can. J. Chemi.-Revue Can. Chim. 1973, 51, 1933-1940.

33. Migron, Y.; Marcus, Y. Polarity and hydrogen-bonding ability of some binary aqueous organic mixtures. J. Chem. Soc.-Faraday Trans. 1991, 87, 1339-1343.

34. Laaksonen, A.; Kusalik, P.G.; Svishchev, I.M. Three-dimensional structure in water-methanol mixtures. J. Phys. Chem. A 1997, 101, 5910-5918.

35. Killmann, H.; Benz, R.; Braun, V. Conversion of the FhuA transport protein into a diffusion channel through the outer membrane of Escherichia coli. EMBO J. 1993, 12, 3007-3016.

36. Nallani, M.; Benito, S.; Onaca, O.; Lindemann, M.; Winterhalter, M.; Meier, W.; Schwaneberg, U. A nanocompartment system (synthosome) designed for biotechnological applications. J. Biotechnol. 2006, 123, 50-59.

37. Prilipov, A.; Phale, P.S.; Van Gelder, P.; Rosenbusch, J.P.; Koebnik, R. Coupling site-directed mutagenesis with high-level expression: Large scale production of mutant porins from E. coli. FEMS Microbiol. Lett. 1998, 163, 65-72.

38. Leammli, U.K. Cleavage of structural proteins during the assembly of the head of bacteriophage T4. Nature 1970, 227, 680-685.

39. Provencher, S.; Glöckner, J. Estimation of globular protein secondary structure from circular dichroism. Biochemistry 1981, 20, 33-37.

40. Deléage, G.; Geourjon, C. An interactive graphic program for calculating the secondary structure content of proteins from circular dichroism. Comput. Appl. Biosci. 1993, 9, 197-199.

(C) 2012 by the authors; licensee MDPI, Basel, Switzerland. This article is an open access article distributed under the terms and conditions of the Creative Commons Attribution license (http://creativecommons.org/licenses/by/3.0/). 